INPLASY

PROTOCOL

To cite: Xu et al. Prognostic and Clinicopathological Significance of TGFBI

Expression in Cancer Patients:

A Meta-Analysis and

Bioinformatics Validation. Inplasy protocol 2021100089. doi:

10.37766/inplasy2021.10.0089

Received: 23 October 2021

Published: 23 October 2021

Corresponding author:

Mingna Xu

1198551962@qq.com

Author Affiliation:

Xuzhou Medical University.

Support: High-level talents project.

Review Stage at time of this submission: Piloting of the study selection process.

Conflicts of interest:

None declared.

\section{Prognostic and Clinicopathological Significance of TGFBI Expression in Cancer Patients: A Meta-Analysis and Bioinformatics Validation}

Xu, MN1; Guo, YR²; Yang, S3; Wang, YR; Liu, GH5.

Review question / Objective: TGFBI was reported to express on various kinds of tumors. Many previous studies have investigated the prognostic significance of TGFBI in cancers.However, the sample number from single study was limited and results remained controversial.Therefore, we performed the frst meta-analysis of the prognostic value of TGFBI expression in diverse malignancies.

Condition being studied: Our analysis was carried out in accordance with Preferred Reporting Items for Systematic reviews and Meta-Analyses (PRISMA) statement. The PubMed, EMBASE, Cochrane library, and Web of Science databases were systematically searched by two authors independently to acquire relevant studies with language restriction to English from January 1, 2000 to June 1, 2021.

INPLASY registration number: This protocol was registered with the International Platform of Registered Systematic Review and Meta-Analysis Protocols (INPLASY) on 23 October 2021 and was last updated on 23 October 2021 (registration number INPLASY2021100089).

\section{INTRODUCTION}

Review question / Objective: TGFBI was reported to express on various kinds of tumors. Many previous studies have investigated the prognostic significance of TGFBI in cancers. However, the sample number from single study was limited and results remained controversial.Therefore, we performed the frst meta-analysis of the prognostic value of TGFBI expression in diverse malignancies.

Condition being studied: Our analysis was carried out in accordance with Preferred Reporting Items for Systematic reviews 
and Meta-Analyses (PRISMA) statement. The PubMed, EMBASE, Cochrane library, and Web of Science databases were systematically searched by two authors independently to acquire relevant studies with language restriction to English from January 1, 2000 to June 1, 2021.

\section{METHODS}

Search strategy: The following retrieval strategy was used: "TGFBI," "cancer," "carcinoma," "tumor," "prognostic," "survival," "prognosis," "outcome," and "mortality." limiting results to studies in humans.

Participant or population: Patients with tumor.

Intervention: High expression TGFBI.

\section{Comparator: Low-expression TGFBI.}

Study designs to be included: (1) Studies were published in English.(2) Studies explored the relationship betweenTGFBI and the prognosis of patients with cancers; (3) The relationships between TGFBI and overall survival (OS) were described; (4) Patients were pathologically diagnosed with any type of human malignancy; (5) The expressions of TGFBI in tumor were measured by immunohistochemical (IHC) staining or quantitative reverse transcriptase polymerase chain reaction (qRT-PCR).(6) Hazard ratio (HR) and 95\% confidence interval $(\mathrm{Cl})$ were reported, or with essential data to calculate it.

Eligibility criteria: (1) Studies were published in English.(2) Studies explored the relationship betweenTGFBI and the prognosis of patients with cancers;(3) The relationships between TGFBI and overall survival (OS) were described; (4) Patients were pathologically diagnosed with any type of human malignancy; (5) The expressions of TGFBI in tumor were measured by immunohistochemical (IHC) staining or quantitative reverse transcriptase polymerase chain reaction (qRT-PCR).(6) Hazard ratio (HR) and 95\% confidence interval $(\mathrm{Cl})$ were reported, or with essential data to calculate it.

Information sources: The PubMed, EMBASE, Cochrane library, and Web of Science databases.

Main outcome(s): Our study revealed that high expression of TGFBI is associated with poor clinical prognosis in cancer patients. TGFBI has the potential to serve as a prognostic marker and a valuable therapeutic target in solid tumors.

\section{Data management: Noteexpress.}

Quality assessment / Risk of bias analysis: Quality of all enrolled literature was evaluated independently by two researchers(Yaru Guo and Song Yang) using the NewcastleOttawa Scale. This quality evaluation scale of literature contains the following three domains: study groups selection, comparison of study groups, and measuring of outcomes. In addition, study with score $\geq 6$ was considered as a high-quality.

Strategy of data synthesis: All p-values were two-sided, and $P<0.05$ was considered statistically significant. Metaanalysis was conducted using the Review manager 5.3 software and STATA software package (version 12.0) (Stata Corp LP, College Station, TX, USA).

Subgroup analysis: Subgroup analyses of OS were further conducted to explore the source of heterogeneity by source of HRs (HRs obtained directly and indirectly), type of cancer(non-digestive system carcinoma and digestive system carcinoma), sample size $(\geq 100$ and $<100)$, factors of region (western country and eastern country), detection method (IHC and non-IHC ).

Sensitivity analysis: According to the available information, we executed subgroup analyses to analyze the possible sources of heterogeneity. The above results suggested that these factors (type of cancer, sample size, factors of region) could explain the potential heterogeneity sources. 
Language: English.

Country(ies) involved: China.

Keywords: TGFBI, tumor, overall survival, prognosis, meta-analysis, bioinformatics analysis.

Contributions of each author:

Author 1 - Mingna Xu.

Email: 1198551962@qq.com

Author 2 - Yaru Guo.

Email: 1768675516@qq.com

Author 3 - Song Yang. 